\title{
FIELD OF POTENTIAL IN A COMPOUND RECTANGLE CONTAINING A LINEAR INCLUSION
}

\author{
BY \\ YU. A. MELNIKOV (Dept. of Mathematical Sciences, \\ Middle Tennessee State Univ., Murfreesboro, TN), \\ V. V. LOBODA (Department of Theoretical Mechanics, Dniepropetrovsk State University, Ukraine), \\ AND \\ V. B. GOVORUKHA (Department of Theoretical \\ Mechanics, Dniepropetrovsk State University, Ukraine)
}

A boundary-value problem in the theory of potential for the compound domain composed of two homogeneous rectangles is solved by the Green's function method. One of the rectangles contains a linear inclusion that is perpendicular to the interface, and whose tip contacts the interfacing line. The integrals (potentials) over the segment of the inclusion are utilized in the solution algorithm. The kernels in these potentials are constructed from the entries in the corresponding matrix of Green's type. In addition, the weakly singular integral equation that results from the formulation of the problem is analyzed. Numerical results demonstrate the accuracy and efficiency of the approach.

1. Introduction. One should recall the importance of Green's functions and matrices in the theory of boundary-value problems in partial differential equations and systems of elliptic type. The Green's function can also be utilized for computing the solution of the problem, once a useful expression for it is available. Because of the lack of useful representations for them, Green's functions are rarely used for these computations. In fact, construction of the Green's function or matrix is cumbersome for even the simplest problems. These constructions are done in closed form for only a few well-known cases. One of these well-known cases is the Dirichlet problem for the two-dimensional Laplace's equation on a simply connected domain. The Green's function for this problem can be written in the form

$$
G(z, \zeta)=\frac{1}{2 \pi} \ln \left|\frac{1-w(z) w(\bar{\zeta})}{w(z)-w(\zeta)}\right|
$$

The points $z=x+i y$ and $\zeta=\xi+i \eta$ are usually called the "field" ("observation") point and the "source" point, respectively. The bar on $\zeta$ indicates the complex conjugate, and the function $w(z)$ maps in a one-to-one conformal manner the given domain $\Omega$ onto the interior of the unit circle. If $w(z)$ can be represented with a finite combination of

Received August 17, 1994.

1991 Mathematics Subject Classification. Primary 35J25, 73C35. 
elementary functions, then Eq. (1) will appear in a closed form. Otherwise, this equation would provide an approximate analytical expression for the desired Green's function. Hence, even for the rectangular domain, one cannot use Eq. (1) to obtain an exact representation for the Green's function of the Dirichlet problem for Laplace's equation because the associated mapping function $w(z)$ is not expressible in closed form. Despite its limitations, Eq. (1) is useful for approximating values of the Green's functions for some domains when numerical procedures are employed for the conformal mapping.

Another practical way to determine the Green's function is the image (reflection) method. The following example illustrates the essence of this approach. Let $G(z, \zeta)$ represent the Green's function of the Dirichlet problem for the infinite strip $\Omega_{S}\{0 \leq$ $\operatorname{Im} z \leq b\}$. Consider also the sum $G^{*}(z, \zeta)=G(z, \zeta)+G(z,-\zeta)$, with $z$ and $\zeta$ being specified in the semi-strip $\Omega_{S S}\{0 \leq \operatorname{Re} z<\infty, 0 \leq \operatorname{Im} z \leq b\}$. Clearly, $G^{*}(z, \zeta)$ represents the Green's function to Laplace's equation over $\Omega_{S S}$ for the specific boundaryvalue problem with Neumann boundary conditions being prescribed along the boundary line $x=0$, and Dirichlet conditions prescribed along the lines $y=0$ and $y=b$. The reflection method is obviously not appropriate either for domains with complicated shapes or simply-shaped domains with mixed boundary conditions. However, this method is helpful in some situations in which either Dirichlet or Neumann conditions are prescribed on the boundary of a simply-shaped domain.

Another well-known technique for constructing Green's functions is based on Fourier's method of separation of variables. This approach is especially applicable to the Dirichlet problem over a rectangular domain. For a rectangle of sides $a$ and $b$, an expression for the Green's function is known in the form

$$
G(x, y ; \xi, \eta)=-\frac{4 a b}{\pi^{2}} \sum_{m, n=1}^{\infty} \frac{\sin \frac{m \pi x}{a} \sin \frac{m \pi \xi}{a} \sin \frac{n \pi y}{b} \sin \frac{n \pi \eta}{b}}{(m b)^{2}+(n a)^{2}}
$$

Although this expression is widely used in mathematical physics, it is not often feasible for computational applications. Indeed, the series in Eq. (2) does not converge uniformly because of the logarithmic nature of its singularity. Some special analytic treatment to accelerate the convergence of the series would have to be applied to Eq. (2) to make it useful.

A more useful alternative for the numerical evaluation of a Green's function for the two-dimensional Laplace's equation can be directly developed from the analytical representation

$$
G(z, \zeta)=\frac{1}{2 \pi} \ln \frac{1}{|z-\zeta|}+g(z, \zeta)
$$

which expresses the Green's function in terms of its logarithmically singular and regular $g(z, \zeta)$ components. Using this expression for computing values of the Green's function leads to a corresponding one-parameter set of boundary-value problems for the regular term $g(z, \zeta)$, with $\zeta$ being the parameter. These computations, as a result, can be very time-consuming. However, this approach can be readily applied to various boundaryvalue problems for other equations and systems of the elliptic type whose fundamental solutions are already known. 
Another approach to computing Green's functions, for specific types of partial differential equations, is provided in [1]. It is based on the second Green's formula, which expresses the solution of a given boundary-value problem in terms of its boundary values, and on the derivation of the Green's function from the solution of an integral equation. This idea is applied to a certain class of equations in [2], an excellent paper which also discusses two methods of solving the basic integral equation, the series and sequence methods. This approach to constructing Green's functions is versatile, but cumbersome.

The use of each of these methods for constructing Green's functions is limited to a specific class of problems. In response to the need for more universal methods, the first of the authors of this paper has proposed $[3,7,8]$ a new technique which was originally directed to mixed boundary-value problems for equations and systems of elliptic type. The technique has proven useful for a variety of problems in applied mechanics. The first step of the approach represents the Green's function to be found by means of its Fourier series with respect to one independent variable. As a result, boundary-value problems for ordinary differential equations arise in the coefficients of the Fourier series. Green's functions for these boundary-value problems are easily obtained. The final phase in our approach deals with either the complete or partial summation of the Fourier expansions. This summation results in an expression of the singularities in the Green's function in terms of elementary functions, with the regular terms expressed by elementary functions or by uniformly convergent series.

This method of deriving the Green's functions can be applied to the analysis of a compound media, one of which is physically or geometrically nonhomogeneous. A compound media consists of several zones, each of which is homogeneous. To extend the definition of the Green's function to the compound media, we consider the compound domain $\Omega=\bigcup \Omega_{i}(i=1, \ldots, m)$ (Figure 1) in two-dimensional Euclidean space. Let the constants $\lambda_{i}$ specify the physical properties of the materials that comprise the homogeneous

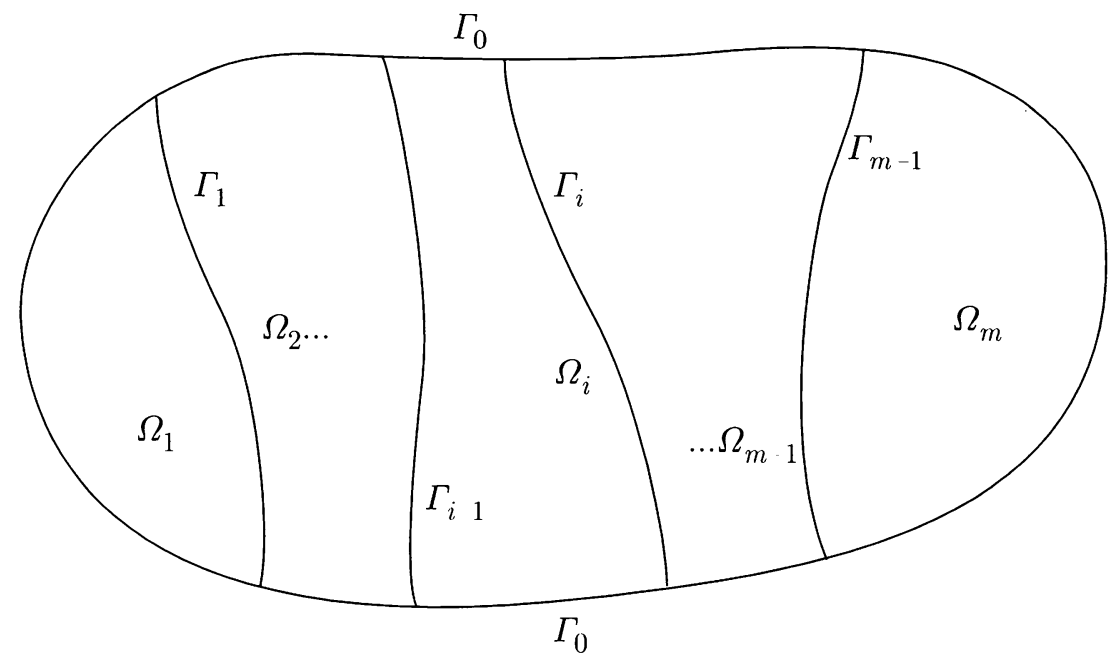

FIGURE 1. Domain $\Omega=\bigcup \Omega_{i}(i=1, \ldots, m)$ of a compound structure 
fragments $\Omega_{i}$. We now state the following boundary-value problem:

$$
\begin{gathered}
\Delta u_{i}(t)=-f_{i}(t), \quad t \in \Omega_{i}, i=1, \ldots, m, \\
u_{i}(t)=0, \quad t \in \Gamma_{0}, i=1, \ldots, m, \\
u_{i}(t)=u_{i+1}(t), \quad t \in \Gamma_{i}, i=1, \ldots, m-1, \\
\lambda_{i} \frac{\partial u_{i}(t)}{\partial n_{i}}=\lambda_{i+1} \frac{\partial u_{i+1}(t)}{\partial n_{i}}, \quad t \in \Gamma_{i}, i=1, \ldots, m-1,
\end{gathered}
$$

where $\Delta$ is Laplace's operator, and $n_{i}$ indicate directions perpendicular to the contact curves $\Gamma_{i}, i=1, \ldots, m-1$.

Assume that the problem above has a unique solution, or equivalently, the corresponding homogeneous problem has only the trivial solution. Consider the vector functions

$$
U(t)=\left[U_{1}(t), \ldots, U_{m}(t)\right], \quad F(t)=\left[F_{1}(t), \ldots, F_{m}(t)\right],
$$

whose components are defined by

$$
U_{i}(t)=\left\{\begin{array}{ll}
u_{i}(t), & t \in \Omega_{i}, \\
0, & t \in \Omega \backslash \Omega_{i},
\end{array} \quad F_{i}(t)= \begin{cases}f_{i}(t), & t \in \Omega_{i}, \\
0, & t \in \Omega \backslash \Omega_{i}\end{cases}\right.
$$

We are now in a position to extend the classical concept of the Green's function to the compound medium.

Definition. For any allowable vector function $\mathbf{F}(t)$, if the vector function $\mathbf{U}(t)$ can be represented by the integral

$$
\mathbf{U}(t)=\iint_{\Omega} G(t, \tau) \mathbf{F}(\tau) d \Omega(\tau)
$$

then the kernel matrix $G(t, \tau)$ in this integral is said to be the matrix of Green's type for the homogeneous boundary-value problem corresponding to the problem stated in (3) $-(6)$.

The entries $G_{i j}(t, \tau)$ in this matrix are defined for $t \in \Omega_{i}$ and $\tau \in \Omega_{j}$ and possess the following properties:

1. $\Delta G_{i j}(t, \tau)=0$, for $t \in \Omega_{i}, t \neq \tau, i=1, \ldots, m$;

2. for $t=\tau, G_{i i}(t, \tau)(i=1,2)$ possesses the logarithmic singularity written as

$$
\frac{1}{2 \pi} \ln \frac{1}{|t-\tau|}
$$

3. $G_{i j}$ satisfies the boundary conditions in (4) and the contact conditions in (5) and (6) for $i=1, \ldots, m$ and $j=1, \ldots, m$.

One of the first applications of matrices of Green's type dealt with some problems in the theory of thin shells, as described in a paper by S. P. Gavelya [4]. He later introduced the term matrix of Green's type, which is used in this paper. 
2. Statement of the problem. Green's function formulation. Consider a rectangular domain $\Omega\{-a \leq x \leq a, 0 \leq y \leq b\}$ consisting of two congruent rectangles $\Omega_{1}\{-a \leq x \leq 0,0 \leq y \leq b\}$ and $\Omega_{2}\{0 \leq x \leq a, 0 \leq y \leq b\}$ (Figure 2). Suppose that $\Omega_{1}$ and $\Omega_{2}$ are composed of materials whose physical properties are specified by the constants $\lambda_{1}$ and $\lambda_{2}$, respectively. Then the corresponding boundary-value problem is given by

$$
\begin{aligned}
\Delta U_{i}(x, y) & =0, & (x, y) \in \Omega_{i} \quad(i=1,2), \\
\left.\frac{\partial U_{1}}{\partial x}\right|_{x=-a} & =0, & \left.U_{2}\right|_{x=a}=0, \\
\left.U_{1}\right|_{y=0, b} & =0, & \left.U_{2}\right|_{y=0, b}=0, \\
\left.U_{1}\right|_{x=0}=\left.U_{2}\right|_{x=0} & =0, & \left.\lambda_{1} \frac{\partial U_{1}}{\partial x}\right|_{x=0}=\left.\lambda_{2} \frac{\partial U_{2}}{\partial x}\right|_{x=0}, \\
\left.U_{2}\right|_{y=y^{*}, 0 \leq x \leq x^{*}} & =C, & (C \text { is a constant }) .
\end{aligned}
$$

Let $G=\left[G_{i j}(x, y ; \xi, \zeta)\right]_{i, j=1,2}$ represent the matrix of Green's type, which is obtained by using the technique found in [8]. Then the potential representations, written as

$$
\begin{aligned}
& U_{1}(x, y)=\int_{0}^{x^{*}} G_{12}\left(x, y ; \xi, y^{*}\right) \mu(\xi) d \xi, \quad(x, y) \in \Omega_{1} \\
& U_{2}(x, y)=\int_{0}^{x^{*}} G_{22}\left(x, y ; \xi, y^{*}\right) \mu(\xi) d \xi, \quad(x, y) \in \Omega_{2}
\end{aligned}
$$

can be used to find a solution to the boundary-value problem in (8)-(12). Indeed, these potential representations satisfy the relations in (8)-(11) for any value of the density function $\mu(\xi)$, which should be integrable on $\left(0, x^{*}\right)$. The entries $G_{12}$ and $G_{22}$ in the

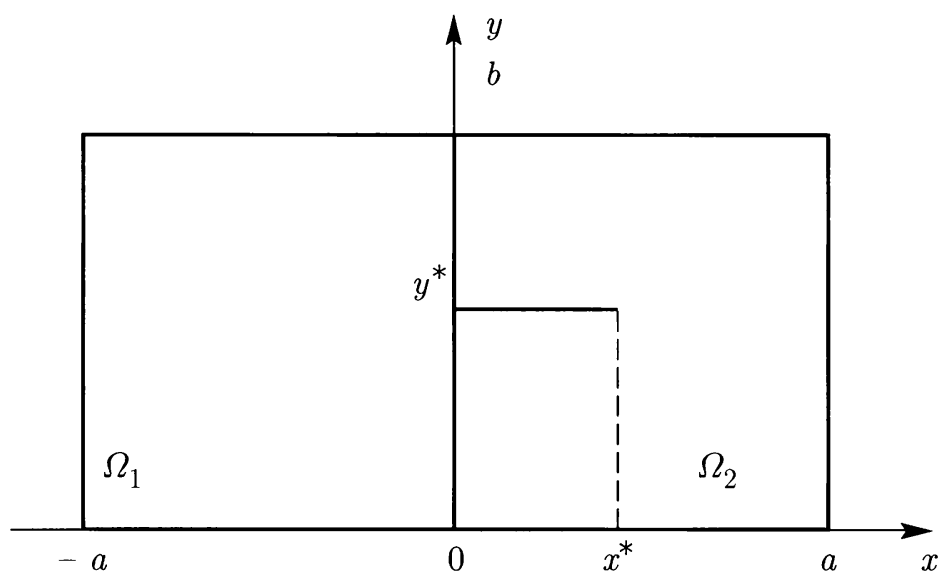

Figure 2. Compound rectangle $\Omega=\Omega_{1} \cup \Omega_{2}$ containing a linear inclusion 
matrix of Green's type are expressed as follows:

$$
\begin{aligned}
G_{12}\left(x, y ; \xi, y^{*}\right)= & \frac{2}{b} \sum_{n=1}^{\infty} \frac{\lambda \cosh \nu(a+x) \sinh \nu(a-\xi)}{\nu \Delta^{*}} \sin \nu y \sin \nu y^{*} \\
G_{22}\left(x, y ; \xi, y^{*}\right)= & \frac{1}{4 \pi}\left[\ln \frac{A\left(x, y ; \xi, y^{*}\right)}{E(z-\zeta)}-\frac{1-\lambda}{1+\lambda} \ln \frac{B\left(x, y ; \xi, y^{*}\right)}{E(z+\bar{\zeta})}\right] \\
& +\frac{2}{b} \sum_{n=1}^{\infty} S_{n}(x, \xi) \sin \nu y \sin \nu y^{*}
\end{aligned}
$$

where

$$
\nu=\frac{n \pi}{b}, \quad \lambda=\frac{\lambda_{2}}{\lambda_{1}}, \quad \Delta^{*}=\sinh ^{2} \nu a+\lambda \cosh ^{2} \nu a,
$$

while the complex variables $z$ and $\zeta$ are given by

$$
z=x+i y, \quad \zeta=\xi+i y^{*} .
$$

A real-valued function $E(t)$ of a complex variable $t$ is defined as

$$
E(t)=\left|1-e^{\pi t / b}\right|
$$

while $A=A\left(x, y ; \xi, y^{*}\right)$ and $B=B\left(x, y ; \xi, y^{*}\right)$ are defined by

$$
\begin{array}{cl}
A=\frac{E(z-\bar{\zeta}) E\left(z-\zeta_{1}\right) E\left(z+\zeta_{2}\right) E\left(z+\bar{\zeta}_{3}\right)}{E\left(z-\bar{\zeta}_{1}\right) E\left(z+\bar{\zeta}_{2}\right) E\left(z+\zeta_{3}\right)}, & B=\frac{E(z+\zeta) E\left(z+\bar{\zeta}_{1}\right)}{E\left(z+\zeta_{1}\right)}, \\
\zeta_{1}=\zeta-4 a, \quad \zeta_{2}=\zeta-2 a, & \zeta_{3}=\zeta+2 a .
\end{array}
$$

The coefficient $S_{n}(x, \xi)$ of the series in (14) is given in the form

$$
\begin{gathered}
S_{n}(x, \xi)=\frac{1}{4 \nu \Delta^{*} e^{2 \nu a}}\left[(\sinh \nu(x+\xi)-\sinh \nu(2 a+x-\xi))\left((1+\lambda) e^{-2 \nu a}-2(1-\lambda)\right)\right. \\
\left.-(1-\lambda) e^{2 \nu a} \sinh \nu(x-\xi)+\frac{1}{1+\lambda}(1-\lambda)\left((1+\lambda) e^{-2 \nu a}-2(1-\lambda)\right) \sinh \nu(2 a-x-\xi)\right] .
\end{gathered}
$$

Expressions for the entries in the matrix of Green's type $G\left(x, y ; \xi, y^{*}\right)$ shown above are valid for $x \leq \xi$. For $x \geq \xi, G\left(x, y ; \xi, y^{*}\right)$ is expressible by interchanging $x$ with $\xi$. Applying the condition in Eq. (12) yields the integral equation

$$
\int_{0}^{x^{*}} G_{22}\left(x, y^{*} ; \xi, y^{*}\right) \mu(\xi) d \xi=C, \quad x \in\left[0, x^{*}\right]
$$

To analyze this equation, we rewrite its kernel $G_{22}\left(x, y^{*} ; \xi, y^{*}\right)$ as follows:

$$
G_{22}=\frac{1}{2 \pi}\left[\frac{1-\lambda}{1+\lambda} \ln \left|\frac{\pi(x+\xi)}{b}\right|-\ln \left|\frac{\pi(x-\xi)}{b}\right|+\frac{1}{2} K\left(x, \xi, y^{*}\right)\right],
$$

where

$$
\begin{aligned}
K\left(x, \xi, y^{*}\right)=\ln \left[\frac{(\pi(x-\xi) / b)^{2}}{\left(1-e^{\pi(x-\xi) / b}\right)^{2}} A\left(x, y^{*} ; \xi, y^{*}\right)\right]-\frac{1-\lambda}{1+\lambda} \\
\quad \times \ln \left[\frac{(\pi(x+\xi) / b)^{2}}{\left(1-e^{\pi(x+\xi) / b}\right)^{2}} B\left(x, y^{*} ; \xi, y^{*}\right)\right]+\frac{8 \pi}{b} \sum_{n=1}^{\infty} S_{n}(x, \xi) \sin ^{2} \nu y^{*}
\end{aligned}
$$

is a continuous function throughout the square $\left(0 \leq x \leq x^{*}\right) \times\left(0 \leq \xi \leq x^{*}\right)$. 
3. Solution of the integral equation. The method of solving the integral equation (15) is based on a separate treatment of the logarithmic singularities in its kernel, which are clearly seen by observing the representation in (16). The first of these is a point singularity that occurs when $x=\xi=0$, while the second is a line singularity that occurs along the diagonal $x=\xi$ of the kernel's domain.

We change the scale along the $x$ and $\xi$ directions by using the following substitutions:

$$
x=\frac{x^{*}}{2}(1+t), \quad \xi=\frac{x^{*}}{2}(1+\tau) .
$$

This rescaling reduces the integral equation (15) to the following form:

$$
\int_{-1}^{1}\left[\frac{1-\lambda}{1+\lambda} \ln |2+t+\tau|-\ln |t-\tau|+M\left(t, \tau, y^{*}\right)\right] \mu_{1}(\tau) d \tau=F, \quad t \in[-1,1],
$$

where

$$
\begin{gathered}
M\left(t, \tau, y^{*}\right)=-\frac{2 \lambda}{1+\lambda} \ln \frac{\pi x^{*}}{2 b}+\frac{1}{2} K\left(\frac{x^{*}}{2}(1+t), \frac{x^{*}}{2}(1+\tau), y^{*}\right), \\
\mu_{1}(\tau)=\mu\left(\frac{x^{*}}{2}(1+\tau)\right), \quad F=\frac{4 \pi C}{x^{*}}
\end{gathered}
$$

Differentiating Eq. (17) with respect to $t$, we have

$$
\int_{-1}^{1}\left[\frac{1-\lambda}{1+\lambda} \frac{1}{2+t+\tau}-\frac{1}{t-\tau}+\frac{\partial}{\partial t} M\left(t, \tau, y^{*}\right)\right] \mu_{1}(\tau) d \tau=0, \quad t \in[-1,1]
$$

In addition, we have the integral condition

$$
\int_{-1}^{1}\left[\frac{1-\lambda}{1+\lambda} \ln \left|2+t^{*}+\tau\right|-\ln \left|t^{*}-\tau\right|+M\left(t^{*}, \tau, y^{*}\right)\right] \mu_{1}(\tau) d \tau=F,
$$

where $t^{*} \in(-1,1)$ is an arbitrary, fixed value of the variable $t$.

An algorithm for solving singular integral equations of the type in (18) is described in [5]. The algorithm is based on the method of mechanical quadratures with the zeroes of the Jacobi function of the second kind being used as collocation points. Assuming that the unknown function $\mu_{1}(\tau)$ possesses integrable singularities, we seek a solution of Eq. (18) of the form

$$
\mu_{1}(\tau)=\mu_{1}^{*}(\tau)(1-\tau)^{-\alpha}(1+\tau)^{-\beta},
$$

where $0 \leq \alpha, \beta<1$, and $\mu_{1}^{*}(\tau)$ satisfies the Hölder condition on $[-1,1]$. To find values of the parameters $\alpha$ and $\beta$, we utilize the characteristic equation

$$
\int_{-1}^{1}\left[\frac{1-\lambda}{1+\lambda} \frac{1}{2+t+\tau}-\frac{1}{t-\tau}\right] \sigma(\tau) d \tau=0
$$

associated with Eq. (18). We introduce the piecewise holomorphic function

$$
\Phi(u)=\frac{1}{\pi} \int_{-1}^{1} \frac{\sigma(\tau)}{\tau-u} d \tau
$$


to rewrite Eq. (21) as

$$
\frac{1-\lambda}{1+\lambda} \Phi(-2-t)+\Phi(t)=0
$$

Using the approach described in [9] for estimating Cauchy-type integrals at the limits of the integration, one obtains for $t \rightarrow-1+0$

$$
\begin{gathered}
\left.\Phi(t)\right|_{t \rightarrow-1+0}=\left.\left[\frac{\cot \pi \beta \cdot \mu_{1}^{*}(-1)}{2^{\alpha}(1+t)^{\beta}}+\Phi_{1}^{*}(t)\right]\right|_{t \rightarrow-1+0}, \\
\left.\Phi(-2-t)\right|_{t \rightarrow-1+0}=\left.\left[\frac{\mu_{1}^{*}(-1)}{2^{\alpha}(1+t)^{\beta} \sin \pi \beta}+\Phi_{2}^{*}(t)\right]\right|_{t \rightarrow-1+0},
\end{gathered}
$$

where

$$
\left|\Phi_{j}^{*}(t)\right| \leq C_{j}(1+t)^{-\beta_{0}}, \quad \operatorname{Re} \beta_{0}<\operatorname{Re} \beta \quad(j=1,2) .
$$

Substituting the expressions from (23) into Eq. (22) yields

$$
\frac{\mu_{1}^{*}(-1)}{2^{\alpha}(1+t)^{\beta} \sin \pi \beta}\left(\frac{1-\lambda}{1+\lambda}+\cos \pi \beta\right)=\Phi_{3}^{*}(t) .
$$

As $t \rightarrow-1+0$, function $\Phi_{3}^{*}(t)$ possesses the same properties as $\Phi_{j}^{*}(t)(j=1,2)$. We therefore write an equation that determines parameter $\beta$ in the form

$$
\cos \pi \beta+\frac{1-\lambda}{1+\lambda}=0
$$

For the right endpoint of $[-1,1]$, we similarly obtain the equation $\cos \pi \alpha=0$, which implies that $\alpha=0.5$. The numerical procedure based on the Gauss-Jacobi quadrature formula written in the following form

$$
\int_{-1}^{1} \frac{\varphi(t, \tau)}{(1-\tau)^{\alpha}(1+\tau)^{\beta}} d \tau \approx \sum_{k=1}^{N} A_{k} \varphi\left(t, \tau_{k}\right)
$$

where the $\tau_{k}$ are zeroes of the Jacobi polynomial $P_{N}^{(-\alpha \cdot-\beta)}(\tau)$, is effective for solving the integral equation (18) subject to condition (19).

As shown in [6], the quadrature formula (24) can be successfully applied to integrals with singularities of the kind in Eq. (18) whenever $t=t_{m}(m=1,2, \ldots, N-1)$ coincides with the zeroes of the Jacobi function of the second kind

$$
Q_{N}^{(-\alpha,-\beta)}(t)=\int_{-1}^{1} \frac{P_{N}^{(-\alpha,-\beta)}(\tau)}{(t-\tau)(1-\tau)^{\alpha}(1+\tau)^{\beta}} d \tau
$$


We will now derive a quadrature formula for computing integrals that possess logarithmic singularities. In doing this, consider the integral

$$
I(t)=\int_{-1}^{1} W(\tau) \ln |t-\tau| \sigma(\tau) d \tau, \quad W(\tau)=(1-\tau)^{-\alpha}(1+\tau)^{-\beta}
$$

associated with the integral equation (19). We rewrite the integral above as follows:

$$
I(t)=\int_{-1}^{1} W(\tau) \ln |t-\tau|[\sigma(\tau)-\sigma(t)] d \tau+\sigma(t) I_{0}(t)
$$

where

$$
I_{0}(t)=\int_{-1}^{1} W(\tau) \ln |t-\tau| d \tau .
$$

By using Gauss-Jacobi quadrature, we obtain

$$
I(t) \approx \sum_{k=1}^{N} A_{k} \ln \left|t-\tau_{k}\right|\left[\sigma\left(\tau_{k}\right)-\sigma(t)\right]+\sigma(t) I_{0}(t) .
$$

Representing $\mu_{1}^{*}(t)$ with a Lagrange interpolation polynomial based at the nodes $\tau_{k}$,

$$
\mu_{1}^{*}(t)=\sum_{k=1}^{N} \mu_{1}^{*}\left(\tau_{k}\right) P_{k}(t), \quad P_{k}=\prod_{j=1, j \neq k}^{N} \frac{t-\tau_{j}}{\tau_{k}-\tau_{j}}
$$

we have

$$
I(t) \approx \sum_{k=1}^{N}\left[A_{k} \ln \left|t-\tau_{k}\right|+\omega(t) P_{k}(t)\right] \mu_{1}^{*}\left(\tau_{k}\right),
$$

where the coefficient $\omega(t)$ is given by

$$
\omega(t)=I_{0}(t)-\sum_{k=1}^{N} A_{k} \ln \left|t-\tau_{k}\right| .
$$

Evaluating the integral in Eq. (18) over the set $t_{m}(m=1,2, \ldots, N-1)$ of zeroes of the Jacobi function of the second kind, and subsequently using the Gauss-Jacobi formula (24) and the quadrature formula (27) to evaluate the integral in (19), lead to a system of $N$ linear algebraic equations in $N$ unknowns $\mu_{1}^{*}\left(\tau_{k}\right)$

$$
\begin{gathered}
\sum_{k=1}^{N} A_{k}\left[\frac{1-\lambda}{1+\lambda} \frac{1}{2+t_{m}+\tau_{k}}-\frac{1}{t_{m}-\tau_{k}}+\frac{\partial}{\partial t} M\left(t_{m}, \tau_{k}\right)\right] \mu_{1}^{*}\left(\tau_{k}\right)=0 \\
\sum_{k=1}^{N}\left[A_{k}\left(\frac{1-\lambda}{1+\lambda} \ln \left|2+t^{*}+\tau_{k}\right|+M\left(t^{*}, \tau_{k}\right)-\ln \left|t^{*}-\tau_{k}\right|\right)-\omega\left(t^{*}\right) P_{k}\left(t^{*}\right)\right] \mu_{1}^{*}\left(\tau_{k}\right)=F .
\end{gathered}
$$

After solving this system, the values of $\mu_{1}^{*}\left(\tau_{k}\right)$ are substituted into (20) to approximate $U_{1}(x, y)$ and $U_{2}(x, y)$ at any interior point in $\Omega_{1}$ and $\Omega_{2}$, respectively, by using the potential representations in Eq. (13). 
4. Numerical results. To show the influence of the parameter $N$ on the accuracy of the solution of the system (28), computations were performed for the following values of geometrical and physical parameters: $a=1, b=1, x^{*}=.5, y^{*}=.5$, and $\lambda=.3$. Table 1 exhibits some results of these computations. The values of $\mu_{1}^{*}(-1)$ and $\mu_{1}^{*}(+1)$ shown in the table were computed by polynomial extrapolation. A reasonable agreement has been found in the results of $\mu_{1}^{*}(t)$ computed for various values of $N$. This agreement indicates, in one aspect, the reliability of our computations.

TABLE 1: Approximate solution of the integral equation in (17)

\begin{tabular}{c|r|r|r|r|r}
\hline$N$ & $\tau_{k}$ & $\mu_{1}^{*}\left(\tau_{k}\right)$ & $\mu_{1}^{*}(-1)$ & $\mu_{1}^{*}(1)$ & $\mu_{1}\left(\tau_{k}\right)$ \\
\hline \multirow{2}{*}{7} & -0.952 & 5.308 & 5.310 & 5.442 & 30.137 \\
& -0.429 & 5.365 & & & 6.574 \\
& 0.355 & 5.523 & & & 5.592 \\
& 0.920 & 5.472 & & 5.457 & 12.413 \\
\hline 7 & -0.985 & 5.332 & 5.335 & & 64.979 \\
& -0.804 & 5.308 & & & 11.970 \\
& -0.459 & 5.347 & & & 6.727 \\
& -0.022 & 5.467 & & & 5.490 \\
& 0.420 & 5.532 & & & 5.721 \\
& 0.776 & 5.502 & & & 2.863 \\
& 0.974 & 5.463 & & & 1.415 \\
\hline \multirow{2}{*}{10} & -0.993 & 5.341 & 5.340 & & 18.049 \\
& -0.903 & 5.325 & & & 9.674 \\
& -0.723 & 5.297 & & & 6.802 \\
& -0.471 & 5.346 & & & 5.698 \\
& -0.173 & 5.423 & & & 5.422 \\
& 0.143 & 5.498 & & & 5.777 \\
& 0.445 & 5.531 & & & 10.029 \\
& 0.702 & 5.512 & & & 30.505 \\
\hline
\end{tabular}

Figure 3 depicts the function $\mu_{1}(t)$ for different values of the parameter $\lambda$ (curves 1 , 2 , and 3 relate to $\lambda=1,3$, and 10 , respectively). Looking at the curves, one can see that $\mu_{1}(\tau)$ depends on the value of $\lambda$ only near the endpoint $(t=-1)$ of the inclusion that contacts the interface. This dependence diminishes near the opposite endpoint $(t=+1)$ of the inclusion, and is not affected by the value of the parameter $\lambda$ (at least over the range considered in this study).

The fields of potential computed for the compound rectangle are illustrated in Figs. 4,5 , and 6 (for $\lambda=100,1$, and .01 , respectively). Clearly, the nonhomogeneity of the material has a greater influence on the field when the subdomain containing the inclusion $\left(\Omega_{2}\right)$ is composed of a material whose coefficient of heat conductivity is smaller than that of the other subdomain $\left(\lambda_{2}<\lambda_{1}\right)$. 


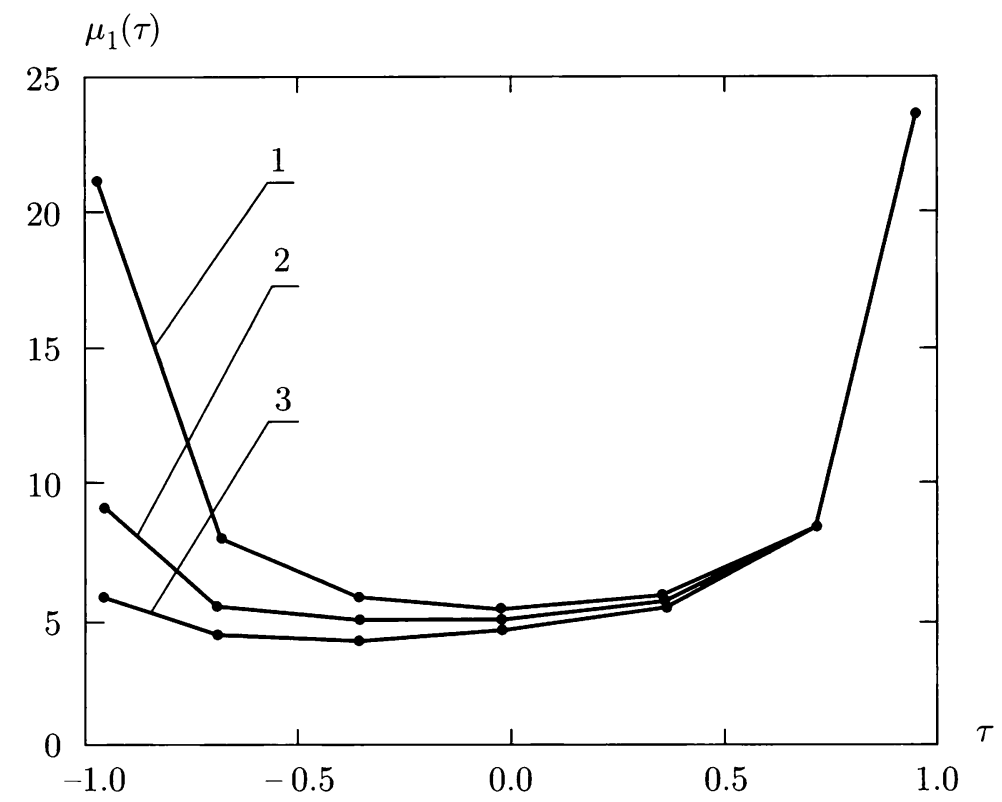

FIGURE 3. Solution of the integral equation (15) for different values of $\lambda$

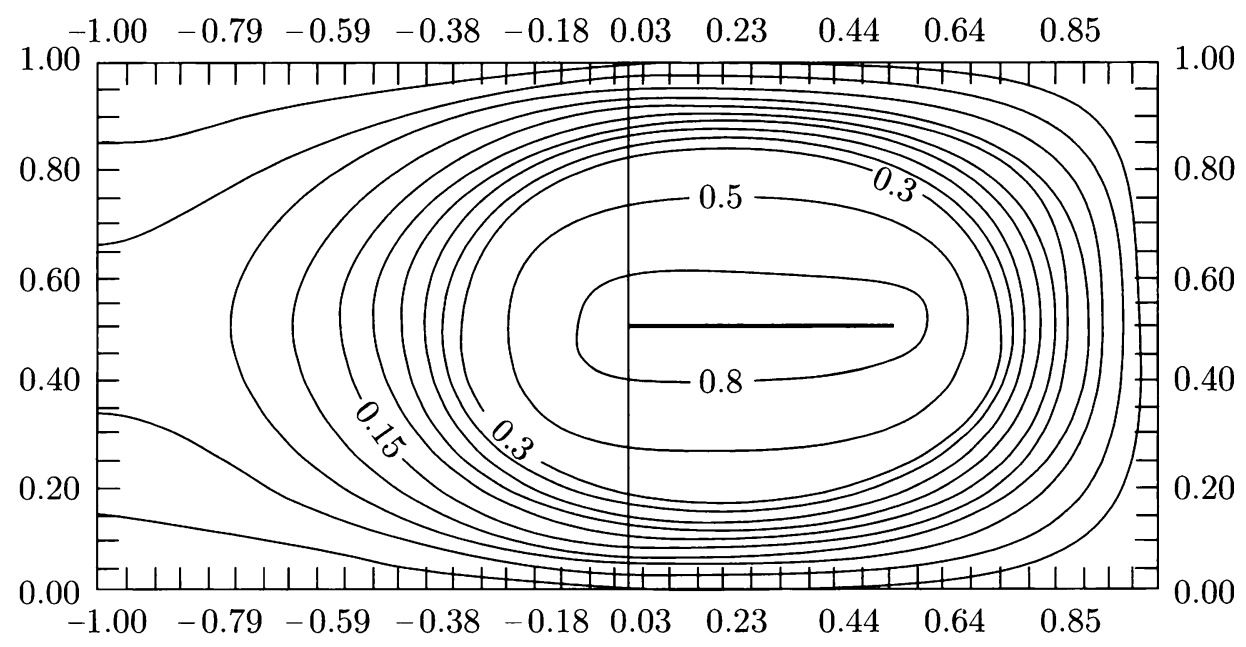

FiguRE 4 . The field of potential in the compound rectangle $(\lambda=$ 100) 


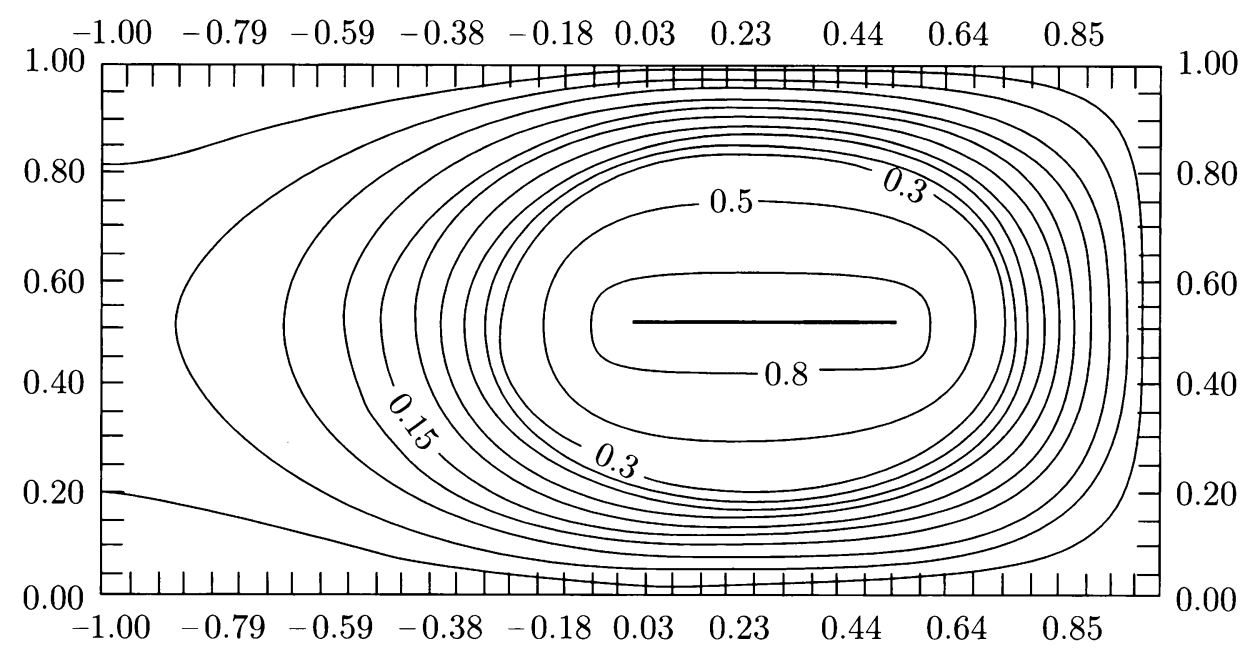

FIGURE 5. The field of potential in the homogeneous rectangle $(\lambda=$ $1.00)$

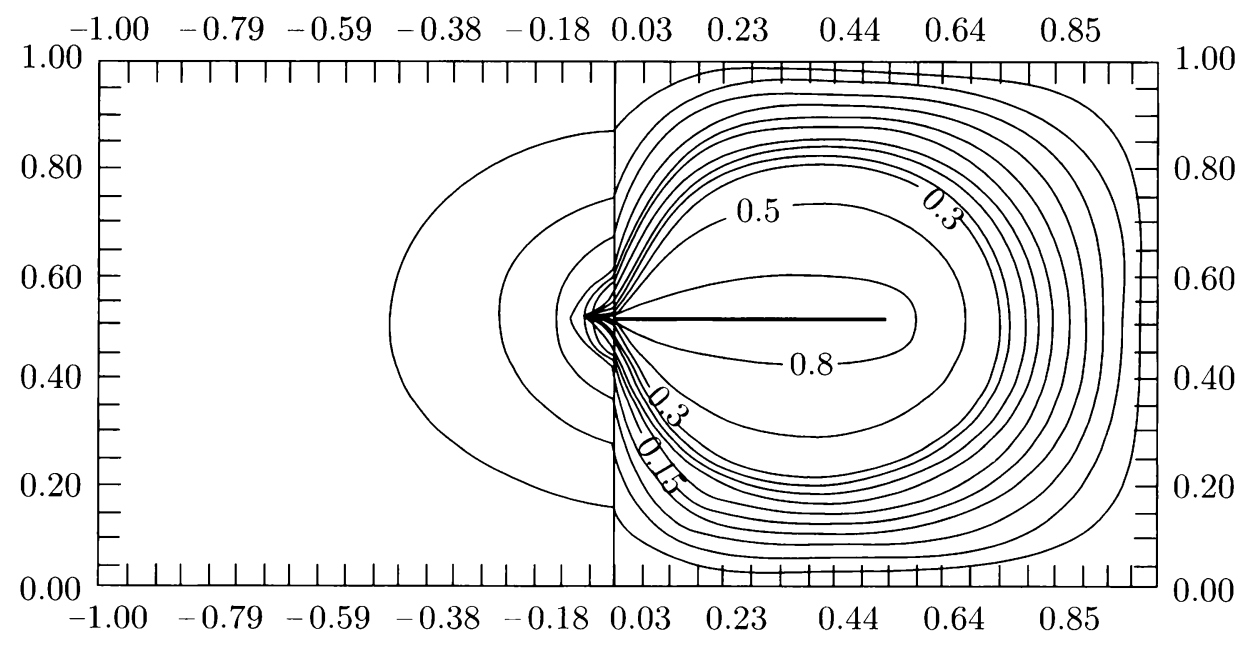

FIGURE 6 . The field of potential in the compound rectangle $(\lambda=.01)$

5. Conclusion. An integral equation method is applied to a compound domain composed of two rectangles, each built of a homogeneous material, and one of which contains a linear inclusion perpendicular to the interface. The Green's function method is adapted for compound domains by extending the definition of the Green's function to a matrix of Green's type. Application of these matrices for constructing kernels in the potential representations is essential to the integral equation method. The densities of the potential representations should be evaluated numerically over the inclusion segment. These potential representations, being harmonic functions (because of a basic property of the matrix of Green's type) in the interior of the domain, satisfy the prescribed set 
of boundary conditions on the contour of the domain and the contact conditions on the interface; this results in line integral equations exclusively along the contour of the inclusion. To numerically solve the line integral equations, we reduce them to a system of linear algebraic equations by using a specially constructed quadrature formula. The order of this system is less than that of more conventional integral equation methods.

This technique is applicable for problems with different boundary conditions on the outer contour of the domain as well as different contact conditions on the line of interface. This versatility is possible because these conditions are treated analytically in the process of constructing the matrix of Green's type. Consequently, the essential parts of the numerical procedure are not affected by a change of either boundary or contact conditions.

Finally, one can compute solutions for various shapes and locations of inclusions, with various kinds of boundary conditions on their contours, simply by reformulating the line integral equations.

Acknowledgment. The first of the authors (Yu. A. M.) wishes to thank Doug Rowe, a graduate student in the Department of Mathematics and Statistics at Middle Tennessee State University for his kind help with the language of this paper.

\section{REFERENCES}

[1] S. Bergman and M. Schiffer, Kernel Functions and Elliptic Differential Equations in Mathematical Physics, Academic Press, Inc., New York, 1953

[2] B. A. Boley, A method for the construction of Green's functions, Quart. J. Appl. Math. 14, 249-257 (1956)

[3] I. M. Dolgova and Yu. A. Melnikov, Construction of Green's functions and matrices for equations and systems of the elliptic type, J. Appl. Math. and Mech. 42, 695-700 (1978) (in Russian)

[4] S. P. Gavelya, On one method of constructing Green's matrices for combined shells, Reports of the Ukrainian Academy of Sciences Ser. A 12, 1107-1111 (1969) (in Russian)

[5] A. A. Korneiytchuk, Quadrature formulas for singular integrals, Numerical Methods for Differential and Integral Equations, and Quadrature Formulas, Nauka, Moscow, 1964, pp. 64-73 (in Russian)

[6] V. V. Loboda, Use of Gauss-Jacobi formula to a solution of singular integral equations, Methods for Solution of Nonlinear Problems and Data Development, Dniepropetrovsk State University Publishers, Dniepropetrovsk, 1986, pp. 60-64 (in Russian)

[7] Yu. A. Melnikov, Some applications of the Green's function method in mechanics, Internat. J. Solids Structures 13, 1045-1058 (1977)

[8] Yu. A. Melnikov, Green's functions and matrices for equations and systems of the elliptic type, Dniepropetrovsk State University Publishers, Dniepropetrovsk, 1991 (in Russian)

[9] N. I. Muskhelishvili, Some Basic Problems in the Mathematical Theory of Elasticity, Nordhoff Ltd., Groningen, 1953 